\title{
Authenticity and Imitation in Translating Exposition: A Corpus-Based Study
}

\author{
Ramadan Ahmed Elmgrab (Corresponding author) \\ Benghazi University, P.O. Box 1308, Benghazi, Libya
}

Tel: 218-928-436-858Ｅmail: ramahmadmegrab@yahoo.com

Received: June 8, 2015 Accepted: June 29, 2015 Published: June 30, 2015

doi:10.5296/jei.v1i1.7781 URL: http://dx.doi.org/10.5296/jei.v1i1.7781

\begin{abstract}
Many Western scholars such as Dryden show little interest in imitations, and express their preference for translations, i.e. paraphrases that are faithful to the sense of the source text. However, they consider imitations as a viable category of translation. It is the degree of freedom, or departure from the original, that differentiates a translation from an imitation. This paper is concerned with issues that are central to the understanding of English-Arabic translation errors when rendering expository text. Not surprisingly, when translating exposition, errors recur especially those relating to the linguistic competence of the students. But not all errors were the same neither was their distribution. Each text-type shows different idiosyncrasies and error distributions which indicate that performance in translation depends largely on the type of text and the rhetorical purposes as well as patterns which follow from the source text. To this end, an error corpus of linguistic structure was collected from the translation project of students majoring in translation. Syntactic, semantic, pragmatic and discoursal criteria were used to judge imitation and authenticity strategies adopted by the students during the translation process. Implications for increasing students' awareness of the pragmatic and syntactic constraints in translating structures will also be provided.
\end{abstract}

Keywords: Translation, Imitation, Authenticity, Corpus, Error analysis, Exposition

\section{Introduction}

Translation has played a key role in the enhancement of communication between peoples of the world. "Translation adds value, bringing other creators and creations into being, in ways that reflect the mobility and multiplicity of our world (Jose, 2015: p. 8). However, the ambivalent role of translation in both establishing and destabilizing notions of authenticity has permeated translation theory. Much of the theoretical discussion on translation has revolved around the idea of equivalence with the original text. To this end, theoreticians strive to find appropriate theories and methods of translation to help translators doing their job. 
Many theories, models and approaches have been proposed and subjected to heated debate amongst theoreticians. In this regard, Elmgrab (2014: p. 131) argues that "different translation models and approaches have sought to eradicate translational misunderstandings, yet each of them has engendered more controversies than solving existing ones". One of these models is text typology which seeks to group texts into categories and types. It aims at identifying and describing linguistic and conceptual features that texts belonging to a particular group have in common. This is because one of the characteristics of text is its resemblance to or difference from other texts. But we may wonder, as did Bell (1991: p. 202) "how is it given that each text is unique, that some texts are treated as the same?" The key concept for answering such a question, he (ibid.) suggests: “... is that of a type-token relationship; each individual text is a token - a realization - of some ideal type which underlies it".

During medieval the Arab scholar Al-Jahiz (Aharoun, 1969: p. 75) had predicted the emergence of the text typological model when he states that "the translator should know the structure of the text, habits of the people and their ways of understanding each other". In the twentieth century, Beaugrande and Dressler (1981: p. 186) hold almost the same notion when they define text-typology as " $a$ set of heuristics for producing, predicting, and processing textual occurrences, and hence acts as a prominent determiner of efficiency, effectiveness, and appropriateness". Both scholars put emphasis on the structure of the text which indicates the rhetorical purpose of the text type, whether it is argumentative, expository or instructive.

Although, the definition of the term text-type varies somewhat between different linguists, most of them follow Hatim and Mason (1990) in relating this concept to communicative intentions. The two scholars (Ibid.:140) define text type as "a conceptual framework which enables us to classify texts in terms of communicative intentions serving an overall rhetorical purpose". In such an approach, texts are defined by features which could be described as external to the text itself. These include areas such as text purpose, text producer's intentions, writer/reader relationships and medium of communication.

The expository text type, our main concern, is used to analyze concepts with the aim of informing or narrating. In this text category, the contextual focus is either on the decomposition (analysis) into constituent elements of given concepts or their composition (synthesis) from constituent elements. There are two important variants of this kind of conceptual exposition differentiated, namely: descriptive and narrative texts. In place of concepts, description handles objects or situations, while narrative texts arrange actions and events in a particular order. Hatim and Mason (1990) postulate that whereas description and narration are generally easily recognizable, boundaries in other cases are more difficult to establish.

I would argue that a kind of conflict could be inferred between the two strategies (imitation and authenticity) in achieving equivalence. On the one hand, authenticity for Pym (2000) may be roughly defined as the opposite of parody, if and when we know what parody is. "Authenticity is the extreme opposite of parody. It is the multiplication of variations beyond anything that the popular imagination can identify" (Ibid.:69). Schleiermacher (Kapsaskis, 2012) located authenticity in the positive act of representing, rather than suppressing, the 
asymmetry that exists between the foreign language and the translator's mother tongue. Berman (Kapsaskis, 2012: p. 1) also concludes that "to authentically represent the foreignness of the original text is a choice that the translator has to make". Authenticity allows receivers to make assumptions about the ST, so to that extent it is source text-oriented. In this respect, Huang (2011: p. 4) argues that "a good literary translation must reproduce something of the source text's style; otherwise the distinguishing literariness in the original will not be conveyed in the target text".

On the other hand, imitation meant a translated text that would present itself as an original. The imitator/translator must give the reader the illusion of reading in the original, and all cultural references must be modified to prevent the reader from feeling altogether out of place. In this regard, Dryden (1680: p. 19) argues that "imitation is not to translate the author's words, or to be confined to his sense, but only to set him as a pattern, and to write, as he supposes that author would have done, had he lived in our age, and in our country". However, for Robinson (1998) imitation is doing something different from the original author, moving freely from the words and the sense of the source language text. Therefore, imitation being considered almost a synonym of free translation, while faithful or authentic translation coincides with the original. In this respect, Fordoński (2014: p. 267) warns:

sometimes, however, translators diverged from so defined a path, pushing the boundaries of imitation so far that instead of moving further to emulate [in the sense of outdoing] an admired author they committed forgeries, altering both the contents and form of the original in a way quite contrary to the author's original intentions.

On the contrary, Sahlin and Wedlin (2008: p. 218) argue that "to imitate, then, is not just to copy, but also to change and innovate". Based on the text typological model, New Mark (2007:14) finds a middle ground between the two strategies. He states that "the more serious and important the language of text, the more closely it should be translated; The less serious and important the language of a text, the less closely it needs be translated". Nevertheless, Newmark favours authenticity when he (Ibid.) maintains that "the better written a text, the closer should be the translation" but he stresses that translation should not be literal, but it should retain the same emphasis of the original and keeping within the bounds of good sense. In this respect, I concur with Hatim and Mason (1990) that the choice of any translation strategy should be determined by the type of text being translated.

\section{Error Assessment}

The study database consists of an English expository text translated by twenty Arab trainee-translators at Benghazi University, Libya into their native language (Arabic). The participants were made under familiar test conditions. They were asked to translate the text, consisting of around 300 words, within a supervised time limit of two hours. Bilingual dictionary was permitted during the performance of the tests. The twenty trainees who undertook the tests were final-year undergraduate students.

As to the analysis of the students' translations, it starts with the recognition of inconsistencies in the text. These inconsistencies are, afterwards, described by locating their linguistic 
realizations. That is, the actual part of the text which bears the inconsistency is defined in terms of the descriptive parameters, i.e. syntactic, semantic and stylistic. The choice of linguistic errors is based on Aixela's (1996: p. 53) assumption that "linguistic codes in themselves are arbitrary systems in which the function of meaning sign's opposition to other signs, and not on a supposed objective relation of equivalence with the continuum we call reality". Errors were explained as to whether they are stimulated by the trainee's lack of competence in the target language (TL) or transfer from the source language (SL).

The contextual focus of the source text (ST) is on the composition (synthesis) of concepts from constituent elements. That is, the author states the topic idea only after presenting a number of sub-topics. For instance, in presenting the topic idea in the text, the author first describes how the principle of necessity is the mother of invention has governed human lifestyles throughout history. The second paragraph shows how the principle finds its way through the development of the study of matter. Only the third paragraph explicitly uncovers the main idea of the text concerning the development of the study of glass. In addition, the text has relatively short sentences which are very condensed in terms of their informational load. The ST involves very few technical words although it seems to present itself as scientific. In terms of general knowledge, it can be said to belong to the overt type and testees are not expected to face difficulties related to culture-specificity.

\subsection{Morpho-Syntactic Errors}

The simpler grammatical structures of the text in hand tend to follow from the rhetorical purposes of its type which often tend to avoid ambiguity, misinterpretation or manipulation of meaning. Morpho-syntactic errors mainly reflect a lack of competence in the syntax of the TL as testees are likely to make the same errors when writing an Arabic essay. On the whole, morpho-syntactic inaccuracy in the translation of exposition was higher because some structures are intricately complex and difficult to understand either in the grammar of English or Arabic. Most errors appear to be typical of individuals rather than the group, i.e. not usually common among all testees. Here, students vary in their translation strategies: some used authenticity and others went for imitation as we shall see in the analysis.

\subsubsection{Tense/Aspect Errors}

Before proceeding with the discussion of errors relating to tense and aspect, it is worth giving a brief definition of the meaning of the two terms in the present context. Both tense and aspect are concerned with correlation between grammatical forms and concepts of time: tense with location in time and aspect with continuing in time. Because of the closeness of the two grammatical forms, errors made in this context will be referred to as tense/aspect errors. This type of error represents in the corpus a high distribution compared with other syntactic errors.

The majority of the students did not follow the English tense system because Arabic is more oriented to aspect rather than tense. Beeston (1970: p. 76) points out in this respect that "very few Arabic verbs embody a wholly unambiguous time signal". Arabic lacks overt (i.e. morphologically indicated) realization of the perfective and progressive, convey just two pragmatic aspects: accomplished (past) or unaccomplished (present). Tenses were incorrectly 
assigned by few students as Sentence 1a below illustrates:

(1) Right from the start he seized what lay around him.

(1a) Haythu annahu yaDa ${ }^{\mathrm{C}}$ yadah $^{\mathrm{C}}$ ala kull ma: Hawlah.

(As he puts his hand on what lay around him).

Some participants used the imperfective in Sentence 1a instead of the past simple as in the original sentence. But the fact that they have rendered all other tense elements correctly suggests the error made in Sentence 1a is not due to the basic incompetence of the student in grammar or tenses. It indicates that the source of failure derived from the construction being translated. Indeed, the adverbial phrase right from the start introducing the sentence is often an indicator of a present perfective in English, and this explains the occurrence of such an erroneous TL construction. One of the students used the appropriate tense but inserted the Arabic complementizer an where none is required as in Sentence 2:

(2) Right from the start he seized what lay around him ...

(2a) munDhu al-bida:ya $a n \mathrm{waDa}^{\mathrm{C}}$ yadah ${ }^{\mathrm{C}}$ ala kull ma: Hawlah ...

(Right from the start, he puts his hand on what lay around him).

The erroneousness of Sentence 2a stems from the fact that the Arabic particle an is usually followed by an expressive verb which must be in the subjunctive mood. The two grammatical conditions are not met in the Arabic translation provided above.

\subsubsection{Agreement and Case-Marking Errors}

Agreement is another area of difficulty where most errors are made individually rather than collectively and tends to reflect the students' lack of competence in Arabic grammar. In fact, case system is a rich but complex syntactic aspect of Arabic which most testees fail at least once to represent appropriately in the text. The difficulty of agreement seems to be related in most cases to gender agreement as in the translation of Sentence 3:

(3) Glass is transparent and made of sand.

(3a) al-zujaj madda shaffafa-wa maSnu ${ }^{\mathrm{C}}$ min alarml.

The students' inability to ascribe the appropriate gender in Sentence 3a is apparently due to their confusion as to whether zuja:j (glass) or ma:dda (matter) is the head noun in the original sentence. This kind of confusion features more in cases like Sentence 3 which involves in Arabic a long nominalized sentence. This type of confusion does not occur in verbal clauses such as in Sentence $3 \mathrm{~b}$ below, where the relation between modifier and modified is clearer:

(3b) li-?anna l-zuja:j yu tabar ma:dda shaffa:fa.

(Because glass is considered a transparent material).

The difficulty with case-marking is greater owing to the complexity of the case system in Arabic. Case-marking is morphologically inflected in Arabic, while it is often uninflected in English. The task is therefore easier in English since it is often the case that exactly the same 
form of a word assumes all different case-markings. As a result, most of the participants followed the English case marking system. However, error of this kind does not affect the intelligibility of the target text (TT) but rather its grammatical acceptability. This reflects the students' difficulty in mastering the Arabic agreement and case system.

\subsubsection{Modality Errors}

The corpus also shows collective errors which can easily pass unnoticed by the translation instructor or evaluator. The translation of the model will into Arabic is a case in point. It is often viewed as a probability model and rendered accordingly into the Arabic futuristic as a probability marker $s a$ - or sawfa (will/shall) as in sawfa takfi or satakfi: (will suffice). In so doing, the trainees sometimes overlook other pragmatic functions that can be realized by the model such as certainty and binding. Almost all testees failed to render the element of certainty embedded in the English expression. As Atari (1994: p. 99) points out, "to render will in Arabic as an expression of certainty about a future, one has to choose the binding universal infinitive": ["takfi:" (suffice) but not "sawfa yakfi (will suffice)" or "sa-yakfi:" (will suffice)]. It can be said that part of the students' problems in rendering English modality is that modality exists as a syntactic as well as a semantic category in English, but only exists as a semantic category in Arabic; hence, the exclusion of the syntactic dimension. As a result, students once again went after the Arabic structure, i.e. they prefer imitation strategy rather than authenticity.

\subsubsection{Negation Errors}

The rendering of the English negation into Arabic can also represent some difficulty to Arab trainees. The students' translations in this context seem to capture the essence of the meaning expressed in the ST. However, other meaning elements which go beyond the logical relations are either absent or misrepresented. Their translations, on the other hand, include aspectual elements which do not exist in the ST because of the wrong selection of the appropriate negation device. Some students used the Arabic negative device laysa (literally: not) which marks a progressive tense aspect, while others used either la: which marks the negation of a habitual present/future action or lam which normally expresses the negation of a past action.

Obviously, tense aspects do not constitute part of the ST and can even hinder the understanding of the original meaning. The negative system in Arabic is more complex than the negation system in English. Therefore, this complexity needs to be highlighted more carefully in Arabic-English translation. Students tried to change English negation devices into their Arabic counterparts but failed to capture the most appropriate meaning.

I asked informally some of the trainees if they noticed any differences between the translations provided in terms of the negation markers. Their answers indicated that most of them knew very well their morphological case-marking, for example that the verb comes after lam must be jussive, but they did not see any difference in terms of meaning. This suggests that trainees often possess a satisfactory knowledge of Arabic prescriptive grammar but they lack the knowledge of the pragmatic functions of grammatical categories, especially functional words. This only emphasizes the fact that translation errors are not a mere 
manifestation of the translation practiced in class but rather it is part of the whole educational culture. Generally, the students' translations of negation seemed to be confusing to the TL readers because of their literalness.

\subsection{Semantic Errors}

The predominant information in the English expository text does not apparently represent significant cross-conceptual differences with that of Arabic. The ideas expressed in the ST are conceptual facts which exist in all time and space regardless of the language they represent (either spoken or written). However, the rendering of concepts into actual words is not always an easy task especially, between different languages. It is expected that trainee students engaged in a (non-professional) general course of translation will encounter difficulties related to their limited lexical and semantic knowledge. This is because English is rich in technical register whereas in Arabic such register is relatively new and, even if it exists, not commonly used. Therefore, a one-to-one correspondence between the SL and the TL word is not always possible.

It is not meant, here, that the translation of a non-technical text like the text in question does not involve many difficulties in finding similar concepts in the TL. There is no absolute similarity between concepts and words and the gap between concepts, cross-linguistically, is expected to be even greater especially if we take into account the fact that "the words of a language often reflect not so much the reality of the world, but the interests of the people who speak it" (Palmer, 1976: p. 21). Therefore, it is likely that something is always lost in the process of translation; loss is the norm rather than the exception. Kussmaul (1995: p. 86) argues, in this regard, that in such cases "the translator has to switch from automatic reflex [authenticity] to reflection, [imitation]. and text analysis comes into play". In their translation, testees not only looked for word-for-word equivalence but also opted, on several occasions, for an idiomatic correspondence whenever it was available in the TL. Yet, when no direct correspondence (at the word or idiomatic level) was available, participants seemed to turn to the bilingual dictionary rather than processing the text as a whole unit for translation. Hence, most of them used authenticity strategy in conveying the meaning.

\subsubsection{Non-Equivalence Type}

The testees' translations do not indicate jargon difficulty given the nature of the text being translated. The semantic field of the text consists of lexical items representing general facts about the world. The text involves difficulties relating to semantic nonequivalence which reflects a deficiency in the TL at the linguistic level rather than the conceptual level. That is, the ST element expresses a concept which exists in the TL but has no lexicalized form. For instance, the translation of the phrasal verb fashion into, provided by most participants, succeeded in conveying the general message of the ST as in Sentence 4:

(4) He seized what lay around him to fashion into tools, with which to sew his way through life.

(4a) wa-istafada min kull ma: hawlah wa-s awwaraha fi adawa:t biwa:siTatiha: shaqqa Tari:qahu fi al-haya:t. 
(He benefited from what lay around him and depicted it into tools with which to sew his way through life).

The translation of Sentence 4a expresses the meaning that tools are a reflection of what lay around man in the stone age which is different from that of the ST, viz. what lay around man (e.g. stones, trees, etc.) was transformed and used as tools. Other translations are either as Shakkal (formed) or yuHawwil (transform), or even as yas $n a^{C}$ (make), all of which lack the appreciative value existed in the SL word. That is, to fashion something into something else refers in English to the making of a work of art, "usually, with one's hands or with only a few tools" (Longman Dictionary, 1989: p. 369).

The translation of the lexical past verb seized is another case in point. The message behind this verb was rendered by all participants, yet loss was inevitable. In English, the concept of seizing, though harsh, can be a legitimate and justified act, as in the text where seizing is justified by the needs of man to hack, carve, and sew his way through life. On the contrary, none of the translations provided by the testees reproduced this meaning of the verb to seize. Most of the translations convey a forceful and illegitimate act. I do not intend here, however, to emphasize the traitorous nature ascribed to the translator by the Italian saying, traduttore traditore (translator is a traitor). Losses, I believe, are inevitable but they can be reduced and even compensated for.

Testees were asked again informally to choose the appropriate Arabic counterpart of the verb seized from the following: istawla (seized with force), istaghalla (exploited), or sakhara (utilized). They maintained that these verbs have almost the same meaning as far as this context is concerned. However, the verb sakhara is really suitable as it captures or at least compensates for both meaning aspects of the ST: first is the harshness of the act given that sakhara connotates with servitude, and second is the righteousness of the act given that it indicates a proper and beneficial use of something. The problem in this regard is that students do not search or question the quality of their translation in order to convey the message of the original. As a result, almost all students chose the first meaning of the English verb from a bilingual dictionary to capture the non-equivalence meaning of the ST.

\subsubsection{Synonymy}

Synonymy errors are not a particularly characteristic of any text type, but the choice between words, phrases or sentences can be problematic when translating an expository text. The translation of exposition usually reflects a state where students are trapped by the limited choice of equivalents provided by a bilingual dictionary. A case in point is the translation of the verb provide in Sentence 5 below:

(5) This metallic glass provides a combination of strength and flexibility nothing else can match.

Testees failed to recognize the different polysemous meanings of the verb provide. They interpreted it as meaning supply and gave the following translations: yuqaddim (present), yuzawwid (supply) and yamnah (offer) which can be considered as hyponyms of the superordinate supply. However, the intended meaning in the ST is that the metallic glass 
represents or constitutes a combination of strength and flexibility. A way of translating Sentence 5 into Arabic is by using the neutral term ${ }^{C}$ iba:ra ${ }^{C}$ an (roughly meaning: as or to be), as in Sentence 5a:

(5a) wa-hadha al-zuja:j al-ma ${ }^{\mathrm{C}}$ dani: ${ }^{C}$ iba:ra ${ }^{C}$ an mazi:j min al-quwwa wal- muru:na.

(This metallic glass is a combination of strength and flexibility).

However, ${ }^{C}$ ib: ara ${ }^{C}$ an lacks the property of evaluativeness existing in the corresponding ST word (provide) which implies novelty and benefit. This can be compensated for by adding expressions such as wa al-jadi:d (literally: and the new thing is) to substitute the missing elements as in Sentence 5b:

(5b) wa aljadi:d anna hadha alzuja:j al-ma ${ }^{\mathrm{C}}$ dani: yushakkil mazi:j min alsala:ba wa almuru:na.

(The new thing is that this metallic glass forms a combination of strength and flexibility).

Most of the students' translations reflect the literal meaning of the ST. The task of the translator, therefore, is not only limited to making the appropriate choice between a set of defined synonyms but also involves interaction with the text and sometimes he has to search for the appropriate meaning far beyond the synonymous choices.

\subsubsection{Collocations}

The meaning of lexical items within collocative expressions is often largely determined by the different genres and types of discourse. The chain of words which constitutes a collocative expression can also determine its meaning, though in most cases the chain of words and discourse are interdependent. For example, most testees translated the expression strength and flexibility as quwwa wa-muru:na which has the same literal meaning as the phrase in the ST. However, the arbitrariness of collocations makes some choices more appropriate than others. In Arabic, when evaluating the strength of a metal we say $\underline{s} a l b$ (solid) rather than qawwi (hard) and the translation salaba wa-muruna (solidity and flexibility) is appropriate in this context.

The same applies to some testees' translations of the expression tendency to shatter. Some of them rendered the expression as naz atahu lil-kasr (its tendency to break). The inappropriateness of this translation is due to the collocation restrictions of the two words kasr (break) and $n a z^{C} a$ (tendency). The noun kasr in Arabic collocates rather with qa:biliyya (literally: predisposition) which is a neutral term whereas $n a z^{C} a$ is evaluative. This is because its selectional restrictions require that it should be accompanied by a word which must be described as [+abstract] and [-socially evaluative] like $n a z^{C}$ atahu lil-sharr (his tendency to do evil things) [+abstract + socially undesirable] and naz ${ }^{C}$ atahu lil-khayr (tendency to do good deeds) [+abstract, + socially/morally desirable]. Collocations seem to have posed on several occasions some sort of difficulty to the students. Consequently, most of them used imitation strategy to determine the collocative meaning of these expressions. 


\subsection{Stylistic Errors}

Translating word-for-word or sentence-for-sentence will not, in most cases, produce an acceptable version of the ST in the TL. Translation, to be successful, must also cater for "the pattern in which the fabric of the text [as a whole] is woven, and the character with which the sentences and structures are stamped" (Buckey, 1994: p. 65). The fabric and character, Buckley tells us about, do not only differ cross-linguistically but also differ within the same language. This is because the discoursal mechanics of text types are not the same. In this regard, Hatim (1989), for instance, claims that in Arabic the verbal clause type tends to predominate in texts whose focus is exposition, whereas the nominal type is a characteristic of texts with an argumentative focus.

\subsubsection{Cohesion}

It is essential that translation students have good knowledge of the mechanics of the language systems they translate into or from. However, the students' translations seem to indicate that their insufficient knowledge of the stylistic fabrics of Arabic made their task hard. For example, the first two sentences taken from the ST's first paragraph:

(6) Man was ever a materialist. Right from the start, he seized what lay around him.

The relation between the two sentences which can be described as a support relation of the category manner is not expressed here by any grammatical cohesive device. In contrast, this relation in Arabic usually requires a grammatical connective and failing to do so results in an unacceptable structure like the translation of sentence $6 \mathrm{a}$ :

(6a) ka:na alinsa:n da?iman ma:ddy mundhu albida:ya kana: yada ${ }^{\mathrm{C}}$ yadah ${ }^{\mathrm{C}}$ ala: kull ma: hawlah.

(Man was ever a materialist from the start, he seized what lay around him).

But being aware of the necessity for a cohesive device does not remove all difficulties. The two clauses in Sentence 6a are rather developmental whereas the relation between the two clauses in the ST is a causal one: the fact that man was a materialistic led him to seize what lay around him. Therefore, the Arabic connective device al-fa: (as/and) would be the most appropriate connector. Accordingly, the most likely translation would read as Sentence 6b:

(6b) kana: al-insa:n da?iman maddi $f a$-mundhu al-bida:ya wada ${ }^{\mathrm{C}}$ yadah ${ }^{\mathrm{C}}$ ala kull ma: hawlah.

(Man was ever a materialist and from the beginning, he seized what lay around him).

Although cohesion is one of the main characteristics which make the whole fabric of the text, its role and forms usually differ according to the demand of each text-type. The structure of Arabic, on the other hand, usually requires specific cohesive devices, no matter how clear and comprehensible the development in the English ST is. "This paratactic form specific to Arabic where clauses are more additive than subordinative" (Johnstone, 1990: p. 217). Most participants went for Arabic structure and added cohesive devices to link the sentences together .Here, the strategy of imitation is dominant in their translation process. 


\subsubsection{Paragraphing}

Paragraphing, in general, is an area of cross-linguistic variation between Arabic and English and it is often thought that Arabic paragraphing presents a problem when translating into English and not vice versa. The most noticeable difference between Arabic and English paragraphing is the way they are linked together. Paragraphing in English makes a new theme or sub-theme while paragraphing in Arabic is mainly used for stylistic reasons, but not generally used as a division of thought or as an independent unit of meaning. Paragraphs in Arabic are strongly tied up to the same theme by way of lexical cohesion. El-Shiyab (1992: p. 319) argues that "common sense dictates that lack of paragraphing of this type in Arabic editorials is a genuine problem for the English reader". The cohesiveness of the Arabic text through lexical repetition is made even heavier by the connectors which accompany paragraphing.

The students' difficulty in using connectives was not confined to sentences but also embraced the linking of paragraphs. As a result, students kept almost the same paragraphing and preserved the same kind of textual cohesion existed in the ST. For example, each paragraph in the ST starts with a new theme without using any linking devices. This attempt to reproduce English replicas in Arabic caused some confusion to Arab readers because they find it difficult to understand the theme text-forms and relations without grammatical connectors. This is because when translating into Arabic, the translator either keeps these thematic units separate in terms of paragraphs as in English and therefore introduces a conceptual framework of text that is alien to Arabic, or restructures the ST according to the Arabic stylistic requirements. The text-typological view of translation can here be complemented by a functional perspective. That is, the translator will have to weigh his choices in relation to the pragmatic function which the text is supposed to fulfill.

\subsubsection{Nominalization}

Among other difficulties testees encountered was the translation of clause type (nominal or verbal). In the original text, the predominance of verbal clause is very clear. In Arabic, however, there is a tendency to use nominal clauses; the reason, Kharma (1983) points out, is that the verb plays a chief role in the structure and meaning conveyed by the English sentence whereas the Arabic verb plays a much more modest part. The verb does not even feature in one type of sentence (nominal/equational sentence) in Arabic. This implies that equivalents for some English sentences may fall within the latter category (i.e. sentences without a verb) and should therefore be translated as such. The analysis of the testees' translations shows that some of them opted for a nominalization process while others preserved the ST structure.

Kharma (Ibid.) states that Arabic nominalized clauses are the equivalent form of the English infinitival ones. It may be the case that infinitival clauses which often assume a subordinate position in English are better translated as nominalized forms in Arabic but certainly this is not the only way for their translation. For instance, the infinitival clause in Sentence 7 is translated successfully as Sentence 7a:

(7) Right from the start he seized what lay around him to fashion it into tools. 
(7a) mundhu al-bida:ya istahwadha al-insa:n ${ }^{\mathrm{C}}$ ala: kull ma: hawlah $w a$ sagahu ${ }^{\mathrm{C}}$ ala: hay?at adawa:t.

(right from the start, man seized everything around him and fashioned it into a form of tools).

Notice that the ST infinitival subordinate clause has been transformed into an inflected additive clause preceded by the coordinative $a l$-wa:w (and) in the TT. This is a case where the students succeeded in keeping the text in line with the rhetorical requirements of the TL. Indeed, the orality nature of Arabic tends to specify for additive rather than subordinative clauses. Sentence 7 a reads more naturally and stylistically effective.

\section{Conclusion}

In this paper, I have attempted to investigate the students' strategies used in translating expository text. I have seen that the source of difficulty in Arabic, as in any other language, lies outside the language as an abstract system. These difficulties are linguistic, semantic and socio-cultural. In most cases students used imitation strategy rather than authenticity in their translations. This means that they sometimes went for the easy option in order to convey the meaning of the original. This is because the more we move away from the ST, the more we are prone to move away from its intended meaning. This does not mean that imitation is not valid, but it is the dominant strategy. It can be used in rendering some parts of the text where there is a linguistic or cultural mismatch between the ST and the TT.

It is true that the students task was not easy because the two languages in question are incongruent linguistically and culturally. However, the analysis of the students' translations shows that whenever there is some similarity between the ST and the TT the students prefer to employ authenticity, and whenever there is cross-linguistic variation they prefer to employ imitation. This can be traced to the fact that the students' main incompetence was the way they processed the ST because they lack the necessary pragma-textual framework and the frame and schema of the type and genre of the text. They made different types of syntactic errors because they pay little attention to the textual aspects of the ST, such as cohesion, coherence and the organization of information. Their inability to construct Arabic grammatical structures correctly is serious and arises more often in certain areas than in others. English structures which do not exist or have no straightforward equivalents in Arabic (e.g. aspect), or structures which are peculiar to Arabic (e.g. case endings), are often a source of confusion for the participants.

In conclusion, the students choice of translation strategies is not based on sound and comprehensive analysis of the ST. They need to discover what it means and what its intentionality is. They seemed to go for a minimal processing of words and sentences and did not give way for a multiplication process within the whole context in order to allow its expressive and communicative aspect to become manifest.

\section{References}

Aharoun, A. (Ed.). (1969). Kita:b Alhayawān [The Book of Animals]. Beirut: Dar Al-kitab Al-Arabi. 
Aixela, J. (1996). Culture-specific Items in Translation. In R. Alvarez \& M. Vidal (Eds.), Translation Power, Subversion (pp. 52-78). Clevedon: Multilingual Matters.

Atari, O. (1994). Semantic/Pragmatic Clause Relations and Modality in English/ ArabicTranslation. Turjumān, 3(2), 91-101.

Beaugrande, R., \& Dressler, W. (1981). Introduction to Text Linguistics. London: Longman.

Beeston, A. (1970). The Arabic Language Today. London: Hutchinson University Library.

Bell, R. (1991). Translation and Translating: Theory and Practice. London: Longman.

Buckey, R. (1994). Style. Turjumān, 3(2), 165-180.

Dryden, J. (1680). Preface to Ovid's Epistles (pp. 68-72). Translated by Several Hands.

Elmgrab, R. (2014). Possible Criteria for Evaluating Students' Translation Errors. International Journal of Humanities and Cultural Studies, 1(3), 131-145.

El-Shiyab, S. (1992). The Structure of Argumentation in Arabic Editorials as a Case Study (Unpublished $\mathrm{PhD}$ thesis). Heriot-Watt University.

Fordoński, F. (2014). Translation - Imitation - Forgery: George Hils and His "Translations" of Maciej Kazimierz Sarbiewski. Face to Face, Page to Page: PASE Papers in Language, Literature and Culture (pp. 276-276). Institute of English: University of Warsaw.

Johnstone, B. (1990). Orality and Discourse Structure in Modern Standard Arabic, In M. Eid \& J. McCarthy (Eds.), Perspective on Arabic Linguistics 2 (pp. 215-233). Amsterdam: Benjamins.

Jose, N. (2015). Translation Plus: On Literary Translation and Creative Writing. A Journal of Literary Translation, 10, 5-17.

Hatim, B. (1989). Text Linguistics in the Didactics of Translation: the Case of the Verbal and Nominal Clause Types in Arabic. International Review of Applied Linguistics, 27(2), 137-144.

Hatim, B., \& Mason, I. (1990). Discourse and the Translator. London: Longman.

Huang, X. (2011). Stylistic Approaches to Literary Translation: With Particular Reference to English-Chinese and Chinese-English translation. Retrieved July 22, 2014, from http://etheses.bham.ac.uk/2949/1/Huang_X_11_PhD.pdf

Kapsaskis, D. (Ed.). (2012). Knowing Oneself, Untranslatably: Paradoxes of Authenticity in an Age of Globalization. Translation and Authenticity in a Global setting. University of Athens.

Kharma, N. (1983). A Contrastive Analysis of the Use of Verb Forms in English and Arabic. Heidelberg: Julius Groos.

Kussmaul, P. (1995). Training the Translator. Amsterdam: Benjamins. 


\section{Macrothink}

Longman Dictionary. (1989). Longman Dictionary of Contemporary English. London: Longman.

Palmer, F. (1976). Semantics. Cambridge: Cambridge University Press.

Pym, A. (2000). Translating Linguistic Variation: Parody and the Creation of Authenticity. In M. A. Vega \& R. Martín-Gaitero (Eds.), Traducción, metrópoliy diáspora (pp. 69-75). Madrid: Universidad Complutense de Madrid.

Robinson, D. (1998). Imitation. In M. Baker (Ed.), Encyclopedia of Translation Studies (pp. 111-112). London: Routledge.

Sahlin, K., \& Wedlin, L. (2008). Ideas, Imitation and Editing. In R. Greenwood, K. Oliver \& R. Suddaby (Eds.), The Sage Handbook of Organizational institutionalism. London: Sage Publications.

\section{Copyright Disclaimer}

Copyright for this article is retained by the author(s), with first publication rights granted to the journal.

This is an open-access article distributed under the terms and conditions of the Creative Commons Attribution license (http://creativecommons.org/licenses/by/3.0/). 